\title{
Gestione del rischio clinico nell'ASL 3 Genovese: esperienze e prospettive
}

\author{
Giovanni Bruno ${ }^{2}$, Angela Corbella ${ }^{2}$, Sara Giacobbe ${ }^{3}$, Bruna Rebagliati $^{4}$
}

Direttore Sanitario ASL 3 Genovese

2 Responsabile Infermieristica Distretto Socio Sanitario n. 8 ASL 3 Genovese. Componente gruppo operativo Unità di Gestione del Rischio (UGR) ASL 3 Genovese

3 Responsabile Infermieristica Ospedali Genova Nord e Distretto Socio Sanitario n. 10 ASL 3 Genovese. Componente gruppo operativo UGR ASL 3 Genovese

4 Coordinatore Presidio Ospedaliero Metropolitano ASL 3 Genovese. Coordinatore UGR ASL 3 Genovese

\begin{abstract}
In recent years all the most authoritative healthcare organizations have underlined the importance of improving clinical risk management strategies to ensure patients safety and cost savings. This article describes a one-year experience in the contest of ASL 3 Genovese, showing the developement of a risk management project, its aims and its limits. An adequate risk management approach should be based on a "system approach", which assumes that errors of individuals can't be avoided, but the system that surrounds them should provide a safety net for these mistakes. Therefore, efforts are to create a cooperation based on trust, where it's possible to learn from errors and thinking together about new solutions.
\end{abstract}

Keywords: risk management, patients safety, healthcare organizations, system approach

Clinical risk management in ASL 3 Genovese: experiences and perspectives

Pratica Medica \& Aspetti Legali 2009; 3(1): 17-26

Se si è realmente motivati a migliorare la qualità, qualsiasi meccanismo sarà efficace. Se viceversa si tratta di mettere in piedi un sistema di facciata, qualsiasi metodo, anche il più sofisticato, sarà destinato a fallire.

Avedis Donabedian

\section{PREMESSA: IL CONTESTO DELLA ASL 3 GENOVESE}

L'ASL 3 Genovese copre un territorio di circa 1.060 $\mathrm{km}^{2}$ che si estende dalla fascia costiera fino all'entroterra e ai confini con il Piemonte, contando, al suo interno circa 40 comuni e 103 strutture territoriali. È una delle ASL più vaste del nostro Pae- se, avendo una popolazione di riferimento pari a 772.237 abitanti, suddivisi ulteriormente in 6 distretti socio-sanitari, comprendenti, ciascuno, tra 102.555 e 163.080 abitanti. Conta, inoltre, 2 RSA (Residenze Sanitarie Assistenziali) anziani, 4 RSA psichiatriche, 2 comunità terapeutiche, 7 centri diurni e 6 ospedali.

La ASL sta vivendo una delicata fase di transizione caratterizzata dalla ridefinizione dell'assetto organizzativo sia della rete ospedaliera, con riconversioni di posti letto e accorpamenti di Unità Operative (UU.OO.) e Stabilimenti Ospedalieri, sia del territorio, in un'ottica di potenziamento e maggior coordinamento/integrazione delle cure primarie che trovano collocazione nei distretti socio-sanitari. Nel nuovo assetto la rete ospedaliera è così formata:

- 1 presidio ospedaliero metropolitano che include i 6 stabilimenti ospedalieri (uno dei 
quali è stato ricompreso all'interno della ASL nel luglio 2008 a seguito di una procedura regionale di de-aziendalizzazione);

- 645 posti letto per pazienti acuti a ciclo completo;

- 157 posti Day Hospital e Day Surgery;

- 94 posti letto con funzione riabilitativa;

- 25 culle;

- 23 posti letto casa di salute (attivata dal 2002 per l'esercizio della libera professione intramuraria);

- 4 servizi psichiatrici di diagnosi e cura;

- 2 servizi di dialisi con 33 postazioni attrezzate complessive.

L'Unità di Gestione del Rischio (UGR) dell'ASL 3 Genovese è stata istituita con deliberazione n. 566 del 23/05/2007 del Direttore Generale, con l'intento di perseguire le seguenti finalità:

- garantire maggiore sicurezza del/al paziente e degli/agli operatori;

- monitorare in modo sistemico gli eventi avversi, riconoscerne le cause e pianificare azioni correttive;

- individuare e revisionare i processi aziendali riconosciuti come a maggior rischio dalla letteratura sull'argomento, individuandone fasi critiche e possibili azioni preventive;

- promuovere e diffondere la "cultura del rischio/della sicurezza” tra i professionisti che operano in azienda (cultura incentrata su una logica orientata alla prevenzione e fondata sulla convinzione che gli errori rappresentano, se adeguatamente analizzati, preziose opportunità di apprendimento) attraverso lo sviluppo e il monitoraggio di iniziative formative per la gestione del rischio clinico;

- coinvolgere e responsabilizzare i professionisti, garantendo anche un feedback sui progetti in corso;

- migliorare i rapporti con l'utenza e l'immagine aziendale;

- ridurre il contenzioso legale;

- contenere i costi assicurativi.

\section{QUADRO CONCETTUALE E METODOLOGICO DI RIFERIMENTO}

Le attività implementate e programmate si sono ispirate ai seguenti principi che verranno brevemente analizzati: 1. Approccio sistemico e clini- co alla gestione del rischio; 2 . Approccio sistemico all'errore; 3 . Coinvolgimento degli operatori e valorizzazione della loro esperienza.

1. Approccio sistemico e clinico alla gestione del rischio. Esiste un consenso pressoché unanime in letteratura relativamente a tale esigenza; come affermava l'Institute of Medicine (IOM) fin dal 1999 nel rapporto "To err is human": "la natura decentralizzata e frammentata del sistema di assistenza sanitaria (alcuni direbbero "non sistema") contribuisce alle condizioni di insicurezza del paziente e rappresenta un ostacolo agli sforzi per migliorare la sicurezza. È necessario un approccio olistico e sistemico che non può focalizzarsi su una singola soluzione» [1]. All'interno di tale visione sistemica si rende necessario individuare le coordinate concettuali relative alla tematica in questione così sintetizzabili:

- sicurezza del paziente, degli operatori e degli ambienti;

- qualità dell'assistenza essendo la sicurezza una delle dimensioni della qualità dell'assistenza;

- Clinical Governance (CG) termine "ombrello" che sottende un approccio innovativo alla qualità dell'assistenza mirante a coordinare/integrare in un'unica strategia gli approcci frammentati oggi prevalenti. Oltre alle tradizionali dimensioni della qualità (efficacia, efficienza, appropriatezza, sicurezza, equità, partecipazione dei cittadini), la Clinical Governance include l'Audit Clinico, l'Evidence Based Practice, la formazione/ricerca e la gestione del personale evidenziando, con quest'ultimo aspetto, la stretta correlazione tra miglioramento della qualità e organizzazione del lavoro. L'approccio utilizzato, in sintesi, può essere definito di tipo "clinico" volto ad aumentare la sicurezza per il paziente e migliorare la qualità dell'assistenza; mentre quello di tipo "assicurativo" si pone la finalità di ridurre il contenzioso per diminuire i costi dei risarcimenti [2].

2. Approccio sistemico all'errore. Sulla base della teoria formulata da Reason occorre partire dal presupposto che gli errori sono inevitabili e che nella maggior parte dei casi gli esseri umani commettono errori perché i sistemi e i processi in cui lavorano sono disegnati/organizzati in maniera inadeguata [3]. Come afferma De Vries nella recente revisione sistematica: «negli anni recenti nella gestione degli Eventi Avversi (EA) si è passati da un approccio centrato sulla persona (incolpare gli individui per gli errori commessi), a un approccio centrato sul sistema. Tale approccio presuppone che le persone commetteranno errori e che il sistema che li circonda deve fornire una 
rete di sicurezza per ridurre questi errori. Di conseguenza gli sforzi per eliminare gli EA devono essere rivolti ad uno specifico sistema. Il nuovo approccio ha spostato il focus del dibattito sugli EA dalle conseguenze legali associate alla responsabilità personale ad un punto di vista maggiormente costruttivo aprendo la strada a ripensare le soluzioni» [4];

3. Coinvolgimento degli operatori e valorizzazione della loro esperienza. Coerentemente con la prospettiva volta al miglioramento della qualità, si è ricercato il massimo coinvolgimento possibile degli operatori con la finalità ultima a medio-lungo termine di favorire una nuova cultura della "sicurezza" e più in generale del miglioramento della qualità.

In linea con il piano concettuale sopra descritto in termini metodologici, abbiamo tenuto conto delle seguenti esigenze:

- la contestualizzazione degli interventi: nell'ottica di approccio sistemico all'errore sopra menzionata e tenuto conto delle peculiarità della nostra ASL abbiamo, fin dall'inizio, attivato linee operative che hanno coinvolto i servizi extra-ospedalieri; si tratta di un aspetto qualificante se si considera che le attività di gestione del rischio sono prevalentemente rivolte all'ambito ospedaliero e/o al paziente "acuto" a fronte di chiare indicazioni normative e di politica sanitaria che mira- no a spostare (e in parte questo processo è già realtà) il baricentro del sistema dall'ospedale al territorio;

- la scelta delle priorità di intervento: in un'ottica di Evidence Based Practice si è tenuto conto da un lato delle raccomandazioni del Ministero della Salute (MDS) e della letteratura, dall'altro dello specifico contesto come evidenziato al punto precedente;

- il coinvolgimento degli operatori, che si è concretizzato con diverse modalità tra cui:

- costituzione di gruppi di lavoro e qualità interdisciplinari, flessibili e aperti ad altri esperti in base al tema trattato;

- progettazione e attivazione di un sito intranet/internet www.gestionerischio.asl3. liguria.it/ con prossima apertura di un forum; sul sito sono disponibili tutte le procedure, la documentazione, le raccomandazioni e i documenti del Ministero della Salute e le linee guida relative alle diverse tematiche correlate al rischio clinico. Da circa tre mesi è stata aggiunta un'altra sezione dal titolo "Echi dalla stampa sul rischio clinico" che mensilmente viene aggiornata con articoli originali e traduzioni di articoli tratti dalla letteratura;

- disponibilità di un indirizzo e-mail di riferimento a cui rivolgersi per qualsiasi problema o proposta;

\begin{tabular}{|c|c|c|}
\hline Tipo di corso & Destinatari & Fase di avanzamento \\
\hline $\begin{array}{l}\text { Corso avanzato di formazione per } \\
\text { formatori ( } 4 \text { giornate) }\end{array}$ & $\begin{array}{l}\text { Componenti UGR e gruppo } \\
\text { operativo UGR }\end{array}$ & Terminato dicembre 2007 \\
\hline $\begin{array}{l}\text { Convegno "Il rischio clinico nella } \\
\text { ASL } 3 \text { Genovese" ( } 1 \text { giornata) }\end{array}$ & Infermieri UU.OO. ospedaliere & Novembre 2007 \\
\hline $\begin{array}{l}\text { Corso base in } 7 \text { edizioni } \\
\text { ( } 1 \text { giornata) }\end{array}$ & $\begin{array}{l}\text { Direttori e coordinatori } \\
\text { infermieristici, tecnici e della } \\
\text { riabilitazione appartenenti a tutte } \\
\text { le UU.OO. ospedaliere e territoriali }\end{array}$ & Terminato maggio 2008 \\
\hline $\begin{array}{l}\text { Corso FAD del Ministero della } \\
\text { Salute in regime di aggiornamento } \\
\text { obbligatorio }\end{array}$ & Medici e infermieri & $\begin{array}{l}\text { Entro anno } 2008 \text { UU.OO. } \\
\text { ospedaliere - Entro anno } 2009 \\
\text { UU.OO. territoriali }\end{array}$ \\
\hline $\begin{array}{l}\text { Corso FAD realizzato a livello } \\
\text { aziendale accreditato ECM } \\
\text { Regione Liguria ( } 20 \text { ore FAD con } \\
\text { test per ogni modulo seguite } \\
\text { da } 2,5 \text { ore d'aula per riepilogo } \\
\text { contenuti e test finale) }\end{array}$ & $\begin{array}{l}\text { Operatori sanitari dell'area } \\
\text { riabilitativa, tecnica e preventiva }\end{array}$ & $\begin{array}{l}\text { Entro giugno } 2009 \text { UU.OO. } \\
\text { ospedaliere - Entro dicembre } 2009 \\
\text { UU.OO. territoriali }\end{array}$ \\
\hline $\begin{array}{l}\text { Incontri di presentazione } \\
\text { "Campagna mani pulite" e } \\
\text { consegna materiale didattico } \\
\text { informativo ( } 2 \text { ore) }\end{array}$ & $\begin{array}{l}\text { Coordinatori infermieristici, tecnici, } \\
\text { della riabilitazione e prevenzione }\end{array}$ & Terminato ottobre 2008 \\
\hline
\end{tabular}

Tabella I. L'articolazione degli interventi formativi

UGR = Unità di Gestione del Rischio; UU.OO. = Unità Operative; FAD = Formazione a Distanza; ECM = Educazione Continua in Medicina 
- organizzazione di riunioni di U.O. su richiesta del personale con particolare riferimento all'applicazione della scheda unica di terapia;

- valorizzazione del ruolo dei coordinatori per la "Campagna mani pulite": supervisione, rilevazione parametri di controllo previsti dalla procedura, sensibilizzazione del personale, gestione del materiale informativo/educativo (manifesti e poster);

- formazione con utilizzo di metodologia didattica attiva (realizzazione di un video didattico dal titolo "Caccia all'errore" nel quale i partecipanti dovevano individuare gli errori ed esercitazione alla compilazione scheda di Incident Reporting sulla base di casi clinici proposti dai docenti);

- coinvolgimento dei coordinatori nella progettazione e realizzazione del corso FAD aziendale rivolto agli operatori sanitari del ruolo tecnico, riabilitativo e preventivo.

\section{INTERVENTI ATTUATI E IN FASE DI ATTUAZIONE}

Sono di seguito descritte le linee di attività che hanno preso avvio dal maggio 2007 (istituzione UGR).

\section{LA FORMAZIONE DEGLI OPERATORI}

L'articolazione degli interventi formativi è sintetizzata nella Tabella I.

\section{LA SEGNALAZIONE SPONTANEA DEGLI EVENTI (INCIDENT REPORTING)}

\section{Premessa}

Partendo dalla considerazione che l'errore è una componente inevitabile della realtà umana, diventa fondamentale riconoscere che anche il sistema può sbagliare, creando le circostanze per il verificarsi di un errore che resta latente fino a quando uno sbaglio dell'operatore non lo rende manifesto.

L'Incident Reporting è la modalità di raccolta volontaria, anche anonima, delle segnalazioni, in modo strutturato, su eventi o quasi-eventi (near miss) indesiderati.

\section{Obiettivo}

Collaudare e validare un sistema di segnalazione spontanea degli eventi avversi e quasi-eventi at- traverso la Scheda di Incident Reporting (GEN-DSMD-scheda Incident Reporting-00).

\section{Interventi}

- Costituzione di un gruppo di lavoro multidisciplinare che ha elaborato, sulla base delle indicazioni regionali e della letteratura, la seguente procedura aziendale: "Il sistema di Incident Reporting (IR). Progetto di sperimentazione in UU.OO. pilota".

- Presentazione della procedura e descrizione degli interventi previsti all'interno del corso base citato in Tabella I; il corso prevedeva un'esercitazione alla compilazione della scheda di IR sulla base di casi clinici proposti dal gruppo docente. A loro volta i direttori e coordinatori che avevano seguito il corso hanno organizzato incontri nelle rispettive UU.OO. al fine illustrare il sistema agli operatori.

- Disponibilità del documento sopra citato e degli allegati sul sito intranet aziendale e internet "Gestione del rischio".

Ad oggi sono giunte all'UGR sette segnalazioni, per ognuna delle quali si è seguito quanto previsto dalla procedura, garantendo un feedback rapido, l'individuazione delle azioni correttive concordate con gli operatori e la valutazione della necessità di estensione delle azioni preventive alle altre UU.OO.

\section{RISCHI CORRELATI AL FARMACO}

\section{Premessa}

«Gli eventi avversi dovuti ad errori in corso di terapia farmacologica sono la causa di danno più frequente nei pazienti ospedalizzati. Uno studio realizzato in 1.116 ospedali statunitensi nel 2001 ha evidenziato che gli errori di terapia si verificano nel $\mathbf{5 \%}$ circa dei pazienti ricoverati in un anno. Questi eventi (Adverse Drug Event, ADE), prevenibili ed evitabili, vanno differenziati dalle reazioni avverse ai farmaci (Adverse Drug Reaction, ADR), legate al farmaco stesso e che vengono rilevate e valutate mediante le attività di farmacovigilanza nazionali, regionali ed aziendali» [5].

\section{Obiettivo}

Migliorare la sicurezza dei pazienti nel processo di gestione del farmaco.

\section{Interventi}

- Costituzione di un gruppo di lavoro multidisciplinare che ha elaborato, sulla base delle 
raccomandazioni ministeriali e della letteratura, le seguenti procedure aziendali:

- "Indicazioni per prescrizione e somministrazione farmaci: le schede unificate per la terapia” [5];

- "Modalità di utilizzo delle soluzioni concentrate di cloruro di potassio ed altre soluzioni concentrate contenenti potassio" [6];

- "Identificazione del paziente" [7];

- "Terapia trasfusionale sicura" [8];

- "Gestione farmaci chemioterapici antiblastici" (in fase di elaborazione).

- Presentazione della procedura e descrizione degli interventi previsti all'interno del corso base citato in Tabella I.

- Disponibilità di tutti i documenti sopra citati sul sito intranet aziendale e internet "Gestione del rischio".

\section{TERAPIA ANTICOAGULANTE ORALE}

\section{Premessa}

Sempre in tema di rischi correlati al farmaco, a livello territoriale è in fase di implementazione il progetto: "Le persone in Terapia Anticoagulante Orale (TAO): revisione e miglioramento del processo di lavoro a livello dei servizi distrettuali". Nei centri prelievi dei 6 distretti della ASL 3 afferiscono circa 3.500-4.000 persone in TAO per il monitoraggio periodico dei valori di INR (International Normalized Ratio, indicatore del tempo di protrombina; media due prelievi/mese $=84.000-96.000$ accessi/anno secondo i dati di dicembre 2007). L'età media dei pazienti in TAO è sostanzialmente omogenea nei 6 distretti: 73 anni per gli utenti ambulatoriali e 82 per quelli domiciliari. Si tratta di una platea di utenti molto numerosa, anziana e di conseguenza con un trend di continuo aumento degli accessi domiciliari (+15\% nell'anno 2006 rapportato al 2005 ; +7\% nell'anno 2007 rapportato al 2006) oltre che di quelli ambulatoriali, dato dall'ampliamento delle indicazioni alla terapia.

\section{Obiettivi}

- Revisionare il processo di lavoro e rafforzare i meccanismi di integrazione e coordinamento tra servizi ospedalieri, MMG (Medici di Medicina Generale), laboratorio analisi e servizi distrettuali.

- Attuare gli interventi educativi e informativi al paziente in TAO e ai familiari.

- Concordare i criteri per il monitoraggio dell'INR al fine di razionalizzare gli accessi e ridurre i tempi di attesa per i prelievi a domicilio.
- Migliorare le modalità di comunicazione dei valori di INR ai pazienti e ai MMG.

\section{Interventi}

Gli interventi già attuati nel corso del 2007 sono:

- corso di formazione rivolto a tutto il personale infermieristico distrettuale: aggiornamento delle conoscenze ed elaborazione prima bozza di un opuscolo educativo e informativo "La tua guida alla Terapia Anticoagulante Orale (TAO)";

- revisione in équipe (medici ospedalieri, referenti MMG, laboratorio analisi e distretti) del processo di lavoro:

- miglioramento della comunicazione tra laboratorio e MMG: invio ai MMG di un report che riporta i valori INR dell'ultimo trimestre/quadrimestre così da facilitare per il MMG una lettura di insieme anziché quella del valore puntuale;

- adozione concordata di indicazioni di massima riportate in letteratura relativamente alla frequenza del prelievo INR per razionalizzare gli accessi;

- condivisione, eventuale revisione e approvazione della bozza di opuscolo informativo da parte della conferenza dei MMG organizzata in ogni distretto per giungere alla stesura definitiva;

- elaborazione di un questionario di gradimento per le persone in TAO che parteciperanno agli incontri di gruppo di educazione terapeutica.

Interventi attuati (2008):

- stampa degli opuscoli (maggio 2008) e diffusione secondo le seguenti modalità:

- incontri di gruppo di educazione terapeutica per tutti gli utenti e familiari (per coloro che effettuano il prelievo a domicilio), afferenti ai centri prelievi distrettuali;

- consegna opuscoli alle UU.OO. ospedaliere della ASL 3 che ricoverano persone già in trattamento con farmaci anticoagulantiorali e/o che iniziano il trattamento in questione;

- consegna opuscoli agli utenti afferenti agli studi dei MMG per la prescrizione di farmaci anticoagulanti orali.

I parametri di controllo dell'intervento educativo che vengono monitorizzati sono:

- $n$. persone in TAO che partecipano agli incontri di educazione terapeutica di gruppo/totale persone in TAO afferenti ai centri prelievi distrettuali;

- risultati questionari di gradimento incontri di educazione terapeutica. 
È in corso di elaborazione la relazione finale relativa al progetto, ma già da ora si può affermare che i primi parziali risultati sono incoraggianti; circa 1.550 persone in TAO (65\% degli invitati nel 2008) hanno partecipato agli incontri di gruppo di educazione terapeutica. Il livello di gradimento degli incontri che si desume da una prima analisi dei questionari è molto elevato.

Nel 2009 proseguiranno gli incontri di gruppo a completamento dell'intervento per i pazienti già in carico, mentre per ogni "nuovo paziente in TAO" sarà programmato un incontro individuale nell'ambito delle attività degli ambulatori infermieristici distrettuali. Sarà inoltre revisionato e ampliato l'opuscolo informativo soprattutto nella parte riguardante la dieta in quanto oggetto di numerose domande e chiarimenti durante gli incontri di educazione terapeutica.

\section{IL RISCHIO DI INFEZIONI ASSOCIATE ALL'ASSISTENZA SANITARIA}

\section{Premessa}

«Le mani pulite sono il singolo fattore più importante nel prevenire la diffusione di patogeni e la resistenza agli antibiotici nelle strutture sanitarie. L'igiene delle mani riduce l'incidenza delle infezioni ospedaliere» [9].

«L'igiene delle mani, un'azione molto semplice, rimane la prima misura per ridurre le infezioni associate alle cure sanitarie e la diffusione della resistenza agli antibiotici» [10]. Nonostante la provata efficacia e la semplicità della misura preventiva, la compliance degli operatori alle raccomandazioni sull'igiene delle mani «rimane estremamente bassa in tutto il mondo. Infermieri e medici generalmente puliscono le mani meno della metà delle volte che dovrebbero. Nei reparti di area critica, dove vi sono ritmi e carichi di lavoro elevati, l'adesione alle buone pratiche può scendere fino al 10\%» [11].

\section{Obiettivo}

Migliorare la compliance degli operatori alle raccomandazioni sull'igiene delle mani al fine di ridurre le infezioni associate all'assistenza sanitaria e socio-sanitaria.

\section{Interventi}

- Costituzione di un gruppo di lavoro multidisciplinare che ha elaborato sulla base delle linee guida di Centers for Disease Control and Prevention (CDC), Organizzazione Mondiale della Sanità (OMS) e documenti MDS la seguente procedura aziendale: "Igiene delle mani”. Gli allegati alla procedura sono:
- "Igiene delle mani: una cornice teorica";

- "Manifesti Ministero della Salute-OMS" sulle indicazioni all'igiene delle mani, tecnica per il lavaggio e frizionamento con soluzione alcolica;

- "Traduzione integrale delle raccomandazioni linee guida CDC".

- Revisione dei consumi di soluzione alcolica prima dell'avvio della campagna con la farmacia ospedaliera e garanzia della disponibilità della soluzione alcolica in tutti i reparti e $\mathrm{i}$ servizi.

- Traduzione dei poster reminders della Campagna del Servizio Sanitario Inglese, NPSA ( $\mathrm{Na}$ tional Patient Safety Agency) "Cleanyourhands Campaign"; si tratta di 25 poster che verranno gestiti dal coordinatore infermieristico dei reparti e servizi ospedalieri e affissi a rotazione per mantenere l'attenzione degli operatori sul problema, oltre che per coinvolgere $i$ pazienti.

- Traduzione delle diapositive dei CDC oltre che della NPSA inglese.

- Stampa dei manifesti Ministero della SaluteOMS e dei poster reminders sopra descritti per tutti i reparti e servizi ASL.

- Organizzazione degli incontri di presentazione "Campagna mani pulite" citati in Tabella I allo scopo di: presentare la procedura e le linee guida CDC con particolare riferimento al frizionamento delle mani con soluzione alcolica; presentare e consegnare il materiale educativo e informativo da utilizzare nell'ambito della "Campagna mani pulite" (Manifesti Ministero della Salute-OMS e poster reminders sopra descritti).

- Disponibilità di tutti i documenti sopra citati sul sito intranet aziendale e internet "Gestione del rischio".

\section{IL RISCHIO DI CADUTA ACCIDENTALE}

\section{Premessa}

Secondo l'OMS «le cadute e i danni ad esse correlati sono una problematica prioritaria per i sistemi sanitari e sociali in Europa e nel mondo, soprattutto tenendo conto del rapido accrescersi dell'aspettativa di vita» [12]. Con l'avanzare dell'età, infatti, aumenta il rischio di caduta e i conseguenti danni. Le cadute provocano un aumento della disabilità, dell'ospedalizzazione e dei decessi.

Il gruppo di lavoro multidisciplinare ha elaborato il progetto "Revisionare la modalità di segnalazione del fenomeno 'cadute' al fine di disporre di dati uniformi, completi e rilevanti atti a individuare fattori di rischio nello specifico contesto aziendale". 


\section{Obiettivi}

- Revisionare la scheda di segnalazione attualmente in uso sulla base delle evidenze riportate in letteratura e dei limiti rilevati durante l'indagine sotto descritta.

- Uniformare le modalità di segnalazione dell'evento "caduta".

- Sensibilizzare gli operatori sanitari sul tema "cadute" e sull'importanza della segnalazione.

- Disporre di una base dati per individuare i fattori di rischio negli specifici contesti aziendali.

- Pianificare futuri interventi mirati di riduzione del rischio.

\section{Interventi}

- Indagine effettuata nel 2007 volta a «quantificare e analizzare il fenomeno cadute in tutti i contesti degenziali della ASL 3 Genovese al fine di disporre di una base dati per avviare un progetto di prevenzione cadute» (periodo considerato 1 gennaio-31 dicembre 2006).

- Elaborazione e diffusione della procedura aziendale "Gestione del paziente caduto e segnalazione dell'evento" volta a migliorare la sorveglianza e il monitoraggio del fenomeno. La procedura, oltre a fornire un breve inquadramento teorico al problema e istruzioni operative per la gestione del paziente caduto, revisiona completamente la modalità di segnalazione. La nuova scheda con voci nella maggior parte dei casi predeterminate viene utilizzata dal $1^{\circ}$ marzo 2008; la scheda è composta da 3 sezioni:

- la prima, a compilazione infermieristica, precisa, oltre ai dati anagrafici, la storia di precedenti cadute, il luogo, la modalità, il meccanismo e la dinamica dell'evento;

- la seconda, a compilazione del medico, evidenzia le conseguenze della caduta, la sede del danno, il tipo di danno, gli accertamenti diagnostici richiesti, i trattamenti terapeutici a breve termine e la terapia farmacologica in atto;

- la terza sezione riporta eventuali interventi di follow-up oltre all'esito degli accertamenti diagnostici effettuati.

- Predisposizione di un apposito software (Mi-

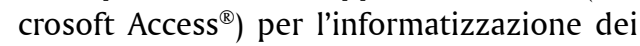
dati contenuti nella scheda da parte delle Direzioni Mediche Ospedaliere e non Ospedaliere e invio contestuale all'Unità Gestione del Rischio (cadenza mensile).

- Presentazione della procedura e descrizione degli interventi previsti all'interno del corso base citato in Tabella I.
- Traduzione delle linee guida NICE (National Institute for Clinical Excellence) "Cadute: la valutazione e la prevenzione delle cadute nelle persone anziane", "Strumenti valutazione rischio caduta”, "Algoritmo di sintesi” 2004 e dell'algoritmo di sintesi "Linee guida per la prevenzione delle cadute nelle persone anziane", American Geriatric Society 2005.

- Disponibilità di tutti i documenti sopra citati sul sito intranet aziendale e internet "Gestione del rischio".

\section{IL RISCHIO DI SUICIDIO}

\section{Premessa}

«Il suicidio in ospedale rappresenta un evento sentinella rilevante, come dimostrato anche dai dati internazionali e, pertanto, è necessario che negli ospedali vengano adottati strumenti e misure idonee di prevenzione» [13].

\section{Obiettivo}

Adottare strategie organizzative e modalità operative atte a ridurre e/o prevenire i suicidi e/o i tentati suicidi nei pazienti ricoverati in strutture ospedaliere e residenziali.

\section{Interventi}

- Costituzione di un gruppo di lavoro multidisciplinare che ha elaborato, sulla base della raccomandazione ministeriale e della letteratura, la seguente procedura aziendale: "Prevenzione del suicidio di paziente ricoverato" [13].

- Presentazione della procedura e descrizione degli interventi previsti all'interno del corso base citato in Tabella I.

- Disponibilità del documento sopra citato sul sito intranet aziendale e internet "Gestione del rischio".

\section{PUNTI DI FORZA E LIMITI DEGLI INTERVENTI ATTUATI}

I punti di forza si possono così sintetizzare:

- coinvolgimento degli operatori e massima diffusione delle informazioni e delle attività dell'UGR (costituzione di gruppi interdisciplinari, sito internet, formazione, interventi su richiesta, "Campagna mani pulite"); 
- attivazione immediata di un sistema di Incident Reporting per favorire la cultura di "apprendere dall'errore";

- individuazione del rischio correlato al processo di gestione del farmaco come priorità di intervento. Come evidenziato infatti dalla recente revisione sistematica sopra citata "gli EA durante il ricovero in ospedale riguardano circa 1 paziente ogni 10 . Una parte considerevole di questi EA sono prevenibili. Poiché la maggior parte degli EA è relativa agli interventi chirurgici e alla terapia farmacologica gli interventi che mirano a prevenire tali eventi sono potenzialmente in grado di fare una sostanziale differenza» [4];

- contestualizzazione degli interventi e coinvolgimento dei servizi extraospedalieri; nel concreto ciò si è tradotto con l'attuazione di interventi rivolti alle persone con problematiche croniche, in prevalenza anziani, che rappresentano la parte più consistente di utenza di una ASL (linea progettuale sulla prevenzione cadute, educazione terapeutica per i pazienti in TAO).

I limiti comprendono innanzi tutto le difficoltà nella revisione del processo di lavoro relativo agli interventi chirurgici, considerato, in letteratura (vedi sopra), un intervento prioritario. Infatti, nonostante la costituzione di un gruppo di lavoro multidisciplinare, non si è ancora giunti a uniformare e proceduralizzare le raccomandazioni del MDS riferite al paziente chirurgico. Le ragioni di tale ritardo sono diverse e interrelate e comprendono:

- la frammentazione delle Sale Operatorie in più Stabilimenti Ospedalieri collocati sul vasto territorio della ASL;

- la notevole disomogeneità nelle modalità e nell'organizzazione del lavoro;

- la mancata conoscenza, da parte degli operatori, di altre realtà e conseguente diffidenza e tendenza all'autoreferenzialità;

- la resistenza al cambiamento.

Vi sono poi difficoltà logistico-strutturali ed economiche per avviare un'informatizzazione del processo di gestione del farmaco (ordinativi e prescrizione informatizzata, dose unitaria ecc.).

\section{PROSPETTIVE FUTURE A BREVE E MEDIO TERMINE}

Le prospettive in tema di gestione del rischio vanno necessariamente inquadrate nella delicata fase di transizione che la ASL sta vivendo, delineata all'inizio dell'articolo. Riteniamo che in un conte- sto in cui gli assetti gestionali, organizzativi e conseguentemente operativi sono in fase di definizione, sia necessario consolidare i progetti già avviati evitando di aggiungere, almeno a breve termine nuove linee di intervento. Si tratta di raccogliere $\mathrm{i}$ frutti dando concretezza a quanto è stato seminato dall'UGR dal maggio 2007 ad oggi.

Analizziamo di seguito quelli che possiamo considerare obiettivi realistici.

\section{LA FORMAZIONE DEGLI OPERATORI}

Completare il piano formativo come programmato.

\section{LA SEGNALAZIONE SPONTANEA DEGLI EVENTI (INCIDENT REPORTING)}

\section{Obiettivi a breve termine}

- Mantenere attivo il sistema garantendo che a ogni segnalazione seguano i relativi interventi da parte dell'UGR.

\section{RISCHI CORRELATI AL FARMACO}

\section{Obiettivi a breve termine}

- Consolidare l'utilizzo della scheda unica di terapia in tutte le UU.OO. verificandone l'applicazione attraverso il parametro di controllo previsto dalla procedura.

- Completare il progetto TAO ed elaborare la relazione finale con gli indicatori previsti dal progetto. Pianificare incontri individuali per ogni nuovo utente che accede al servizio.

- Completare la procedura "Gestione dei farmaci chemioterapici e antiblastici".

\section{Obiettivi a medio termine}

- Attivare l'utilizzo di braccialetti identificativi per i pazienti ricoverati nel Presidio Ospedaliero Metropolitano.

- Elaborare un progetto per avviare l'informatizzazione del processo di gestione farmaco.

\section{IL RISCHIO DI INFEZIONI ASSOCIATE ALL'ASSISTENZA SANITARIA}

\section{Obiettivi a breve termine}

- Monitorare i parametri previsti dalla procedura "Igiene delle mani" e dalla "Campagna 
mani pulite" (monitoraggio consumi soluzione alcolica e sapone, checklist di osservazione nelle UU.OO. per disponibilità dei prodotti: soluzione alcolica e sapone, utilizzo materiale informativo/educativo, tra cui manifesti MDS e poster).

Sarà inoltre mantenuto aggiornato il sito intranet/internet di gestione del rischio e si procederà all'attivazione di un forum così da mantenere sempre aperto il canale di comunicazione con gli operatori.

A medio termine infine e, in considerazione di quanto evidenziato in letteratura, si affronterà la revisione del processo di lavoro relativo agli interventi chirurgici al fine di uniformare i comportamenti di tutte le sale operatorie (sulla base delle due raccomandazioni ministeriali e della letteratura).

\section{IL RISCHIO DI CADUTA ACCIDENTALE}

\section{Obiettivi a breve termine}

- Quantificare il fenomeno "cadute accidentali” nell'anno 2008 utilizzando la nuova scheda di segnalazione.

- Comparare i dati con l'indagine effettuata nel 2006.

- Apportare eventuali modifiche alla scheda.

\section{Obiettivi a medio termine}

- Valutare fattori di rischio specifici.

- Individuare e sperimentare uno strumento di identificazione di pazienti a rischio di caduta.

- Elaborare una procedura di "Prevenzione cadute accidentali” contestualizzata.

\section{CONCLUSIONI}

Il problema della sicurezza dei pazienti è un problema rilevante in termini numerici, economici ed etici. La gestione "strutturata" del Rischio Clinico richiede, innanzitutto, una profonda conoscenza qualitativa e quantitativa delle insufficienze attive e latenti del sistema, degli errori, degli eventi avversi e di quelli evitati.

Una gestione efficace del rischio clinico presuppone che tutto il personale sia a conoscenza del problema, che sia incoraggiata la segnalazione degli eventi e che si presti attenzione ai reclami e al punto di vista dei pazienti.
L'esperienza in atto all'interno della nostra Azienda si propone di aumentare tra gli operatori la consapevolezza del problema "rischio clinico", favorendo la cultura della sicurezza che, anziché colpevolizzare il singolo, considera l'errore come fonte di apprendimento e fenomeno organizzativo.

Occorre creare (ed è il lavoro che stiamo cercando di portare avanti) un clima di fiducia, dove sia davvero possibile imparare dai propri errori attuando i principi della learning organization, da più parti invocati a livello internazionale e non solo in ambito sanitario.

Indispensabile, per il raggiungimento degli obiettivi che ci siamo prefissati, il coinvolgimento dei clinici a tutti i livelli organizzativi del progetto, e in tal senso abbiamo indirizzato le nostre attività. I primi riscontri in merito al sistema di gestione del rischio adottato hanno evidenziato le potenzialità del progetto, soprattutto in relazione alla maggiore consapevolezza, da parte degli operatori, del problema della sicurezza dei pazienti e della grande opportunità di miglioramento offerta dal sistema organizzativo attivato.

In termini strategici sarà opportuno raccogliere la sfida comune che, anche a livello internazionale, è rappresentata dallo sviluppo di misurazioni sistematiche. Come evidenziato infatti dalla recente analisi di Vincent e colleghi, dopo oltre un decennio di sforzi per migliorare la sicurezza «risulta difficile rispondere a questa semplice domanda. "I pazienti sono più sicuri?" Nonostante alcuni aspetti relativi alla sicurezza del paziente siano difficili da misurare per ragioni tecniche (per es. definire la prevenibilità), il problema principale è che la misurazione e la valutazione non sono stati considerati una priorità. Crediamo che la mancanza di informazioni/dati affidabili sulla sicurezza e la qualità delle cure stia ostacolando il miglioramento della sicurezza nel mondo» [14].

Sicuramente il percorso di gestione del rischio clinico richiede impegno, fatica e può partire solo dalla consapevolezza e convinzione profonda che un'assistenza di qualità è un diritto umano fondamentale.

A conclusione di questo resoconto di esperienze con luci e ombre si pone una riflessione sulla sfida della qualità che richiede cambiamenti culturali e valoriali di tutti gli operatori; essa deve diventare uno stile di lavoro basato su competenze aggiornate, un comportamento etico che pone al centro il valore della persona assistita, un modo di relazionarsi con i pazienti e con i colleghi caratterizzato dalla capacità di ascolto e di empatia e soprattutto la normalità dei comportamenti e non qualcosa in più di cui occuparsi a margine dell'attività quotidiana.

Si tratta di un percorso lungo che richiede impegno, fatica e può partire solo dalla consapevolezza e convinzione profonda che un'assistenza di qualità è un diritto umano fondamentale. 
Come sottolinea infatti la Commissione Europea «l'accesso a servizi sanitari di elevata qualità è un diritto umano fondamentale riconosciuto e valorizzato dall'Unione Europea, dalle sue istituzioni e dai cittadini d'Europa. Di conseguenza i pazienti hanno il diritto di aspettarsi che venga compiuto ogni sforzo per garantire la loro sicurezza come fruitori di tutti i servizi sanitari» [15].

\section{BIBLIOGRAFIA}

1. Institute of Medicine. A cura di Kohn LT, Corrigan JM, Donaldson MS. To err is human. Building a safer health system. Washington: National Academy Press, 2000

2. Novaco F, Damen V. La gestione del rischio clinico. Torino: Centro Scientifico Editore, 2004

3. Reason J. Human Error. Cambridge: Cambridge University Press, 2007

4. de Vries EN, Ramrattan MA, Smorenburg SM, Gouma DJ, Boermeester MA. The incidence and nature of in-hospital adverse events: a systematic review. Qual Saf Health Care 2008; 17; 216-23

5. Ministero della Salute. Raccomandazione n. 7, 2007

6. Ministero della Salute. Raccomandazione n. 1, 2005

7. Ministero della Salute. Raccomandazione n. 3, 2006

8. Ministero della Salute. Raccomandazione n. 5, 2007

9. CDC, Boyce JM, Pittet D. Guidelines for hand Hygiene in Health care Settings. MMWR 2002; 51: 1-44

10. World Health Organization. WHO guideline on hand hygiene in health care (advanced draft). Ginevra: WHO, 2006

11. World Health Organization. Global Patient safety challenge. Clean care is safer care. Ginevra: WHO, 2005

12. World Health Organization Europe. What are the main risk factors for falls amongst older people and what are the most effective interventions to prevent these falls? Ginevra: WHO, 2004

13. Ministero della Salute. Raccomandazione n. 4, 2006

14. Vincent C, Aylin P, Franklin BD, Holmes A, Iskander S, Jacklin A et al. Is health care getting safer? BMJ 2008; 337: a2426

15. EAHP. Patient Safety. Making it happen. Luxembourg Declaration on patient safety. EJHP 2005; 4: 69-70

\section{BIBLIOGRAFIA PER APPROFONDIMENTI}

- Bucci R (a cura di). Manuale di Medical Humanities. Milano: Zadig, 2006

- Caminati A, Di Denia P, Mazzoni R (a cura di). Risk management. Manuale teorico-pratico per le professioni dell'assistenza. Roma: Carocci editore, 2007

- Cinotti R (a cura di). La gestione del rischio nelle organizzazioni sanitarie. Roma: Il Pensiero Scientifico Editore, 2004

- Leape Lucian L, Berwick DM. Five years after to err is human. What have we learned? JAMA 2005; 293: 2384-90

- Ministero della Salute, Commissione Tecnica sul Rischio Clinico. Risk Management in Sanità. Il problema degli errori. Caleidoscopio 2003; 199

- Nuti S, Tartaglia R, Niccolai F (a cura di). Rischio clinico e sicurezza del paziente. Modelli e soluzioni nel contesto internazionale. Bologna: Il Mulino, 2007

- Raleigh VS, Cooper J, Bremner SA, Scobie S. Patient safety indicators for England from hospital administrative data: case-control analysis and comparison with US data. BMJ 2008; 337: a1702

- Vincent C. Incident reporting and patient safety. Emphasis is needed on measurement and safety improvement programmes. BMJ 2007; 334: 51 